\title{
Static polarizability of carbon nanotubes: ab initio independent-particle calculations
}

\author{
G.Y. Guo ${ }^{\text {a,* }}$, K.C. Chu ${ }^{\text {a }}$, Ding-sheng Wang ${ }^{b}$, Chun-gang Duan ${ }^{c}$ \\ a Department of Physics, National Taiwan University, 1 Sec. 4, Roosevelt Road, Taipei 10617, ROC, Taiwan \\ $\mathrm{b}$ Institute of Physics, Chinese Academy of science, Beijing 100080, China \\ ${ }^{\mathrm{c}}$ Department of Physics, University of Nebraska-Omaha, Nebraska 68182-0266, USA
}

Received 12 December 2003; received in revised form 24 January 2004; accepted 2 February 2004

\begin{abstract}
An ab initio study of the static polarizability of the carbon nanotubes within density functional theory in local density and independent-particle approximations has been performed. Full-potential projected augmented wave method was used. Specifically, the static dielectric function $\varepsilon(0)$ and electric polarizability $\alpha(0)$ of a number of the armchair $[(3,3),(5,5),(10,10),(15,15),(20,20)]$, zigzag $[(5,0),(10,0),(15,0),(16,0),(20,0)]$ and chiral $[(4,2),(6,2),(6,4),(8,4)$, $(10,5)]$ carbon nanotubes have been calculated. The underlying atomic structure of the carbon nanotubes was determined theoretically. The calculated static polarizability $\alpha(0)$ for the nanotubes is rather anisotropic with $\alpha(0)$ for electric field parallel to the tube axis $(E \| \hat{z})$ being two to three times larger than that for electric field perpendicular to the tube axis $(E \perp \hat{z})$. This anisotropy is further enhanced by up to a factor of five when the depolarization effects for $E \perp \hat{z}$ is taken into account by a classical correction. For both electric field polarizations, $\alpha(0)$ is roughly proportional to the square of the tube diameter, suggesting that it is independent of the chirality and the details of the electronic structure of the nanotubes.
\end{abstract}

(C) 2004 Elsevier B.V. All rights reserved.

Keywords: Carbon nanotubes; Polarizability; Ab initio calculation

\section{Introduction}

Since their discovery in 1991 [1], carbon nanotubes (CNTs) have attracted considerable interest worldwide because of their unusual properties and great potentials for technological applications. For example, because of their one-

\footnotetext{
${ }^{*}$ Corresponding author. Tel.: +886-2-33665180; fax: +886-223639984.

E-mail address: gyguo@phys.ntu.edu.tw (G.Y. Guo).
}

dimensional character, metallic CNTs are quantum wires that may exhibit exotic Luttinger-liquid behavior rather than usual Fermi-liquid behavior in normal metal wires [2]. It was also predicted that nanotori formed from metallic CNTs may exhibit giant paramagnetic moments $[3,4]$. CNTs can be considered as a layer of graphene sheet rolled up into a cylinder, and the structure of a CNT is completely specified by the chiral vector which is given in term of a pair of integers $(n, m)$ [5]. Simple $\pi$-band tight-binding model predicts that depending on the way of the rolling up the 
nanotube can be metallic or semiconducting or insulating [5]. CNTs can be chiral or nonchiral, again depending on the way of the rolling up. CNTs are classified into three types, namely, armchair $(n, n)$ nanotubes, zigzag $(n, 0)$ nanotubes, and chiral $(n, m)$ nanotubes with $n \neq m$ [5]. Because of one-dimensional character and chirality, chiral CNTs are expected to exhibit a number of unusual optical properties such as optical activity, circular dichroism and second harmonic generation [6-9].

Therefore, the optical properties of carbon nanotubes has been intensively investigated theoretically $[6,10,11]$. The static dielectric constants and electric polarizability have also been studied [12] All these theoretical works are, however, based on either tight-binding model or other phenomenological models. Accurate ab initio calculations of the optical properties of CNTs have appeared only very recently $[13,14]$ perhaps because of the heavy demand of the computing resources. Simple $\pi$ band model is known to describe well only the electronic excitations around the Fermi level of the large radius CNTs. Accurate ab initio calculations of the optical properties are thus needed in order to quantitatively interpret optical experiments. We have recently carried out a systematic ab initio study of the optical properties of a number of carbon nanotubes [15]. In this paper, we report the results of our $a b$ initio calculations of the static dielectric constants and electric polarizability for the carbon nanotubes.

\section{Theory and computational method}

Our ab initio calculations for the CNTs were performed using full-potential projected augmented wave (PAW) method [16], as implemented in the VASP package [17]. They are based on density functional theory with local density approximation (LDA). A supercell geometry was adopted so that the nanotubes are aligned in a square array with the closest distance between adjacent nanotubes being at least $6 \AA$. A large plane-wave cut-off of $450 \mathrm{eV}$ was used throughout. We consider a few representative CNTs with a small or moderate diameter from all three types, as listed in Table 1.

Firstly, the ideal nanotubes were constructed by rolling-up a graphene sheet. Their atomic positions and lattice constants were then fully relaxed by a conjugate gradient technique. Theoretical equilibrium nanotube structures were obtained when the forces acting on all the atoms were less than 0.02

Table 1

Calculated radius $R$, band gap $E_{\mathrm{g}}$, static dielectric constant $\varepsilon(0)$ and polarizability $\alpha(0)$ per unit length for the nanotubes studied

\begin{tabular}{|c|c|c|c|c|c|}
\hline & $R(\AA)$ & $E_{\mathrm{g}}\left(E_{\mathrm{g}}^{\mathrm{TB}}\right)(\mathrm{eV})$ & $\varepsilon_{x x}\left(\varepsilon_{z z}\right)$ & $\alpha_{x x}\left(\alpha_{x x}^{\mathrm{cor}}\right)\left(\AA^{2}\right)$ & $\alpha_{z z}\left(\AA^{2}\right)$ \\
\hline$(4,2)$ & 2.12 & $0.26(0.98)$ & $7.0(15.4)$ & $21.5(4.39)$ & 51.6 \\
\hline$(6,2)$ & 2.85 & $0.67(0.70)$ & $7.2(21.9)$ & $29.4(6.41)$ & 99.0 \\
\hline$(6,4)$ & 3.43 & $1.09(0.61)$ & $7.3(22.1)$ & $36.0(8.26)$ & 120.6 \\
\hline$(8,4)$ & 4.15 & $0.81(0.49)$ & 8.7 (23.6) & $53.4(11.3)$ & 156.7 \\
\hline$(10,5)$ & 5.18 & $0.74(0.39)$ & $9.9(25.3)$ & $77.2(16.1)$ & 210.8 \\
\hline$(5,0)$ & 2.02 & $0.0(0.98)$ & $8.5(-)$ & $25.6(4.31)$ & - \\
\hline$(10,0)$ & 3.93 & $0.76(0.49)$ & $8.2(26.8)$ & $47.2(10.2)$ & 169.2 \\
\hline$(15,0)$ & 5.80 & $0.025(0.0)$ & $10.2(-)$ & $90.2(19.3)$ & - \\
\hline$(16,0)$ & 6.26 & $0.18(0.31)$ & $11.0(33.9)$ & $104.9(22.0)$ & 345.2 \\
\hline$(20,0)$ & 7.81 & $0.50(0.24)$ & $13.0(36.1)$ & $156.9(32.2)$ & 459.0 \\
\hline$(3,3)$ & 2.06 & $0.0(0.0)$ & $6.6(-)$ & $19.7(4.18)$ & - \\
\hline$(5,5)$ & 3.39 & $0.0(0.0)$ & $7.7(-)$ & $71.7(9.18)$ & - \\
\hline$(10,10)$ & 6.73 & $0.0(0.0)$ & $11.2(-)$ & $115.5(24.7)$ & - \\
\hline$(15,15)$ & 10.13 & $0.0(0.0)$ & $14.7(-)$ & $232.2(50.3)$ & - \\
\hline$(20,20)$ & 13.48 & $0.0(0.0)$ & $17.8(-)$ & $380.4(84.0)$ & - \\
\hline
\end{tabular}

$\overline{E_{\mathrm{g}}^{\mathrm{TB}}}$ and $\alpha_{x x}^{\text {cor }}$ denote the band gap predicted by $\pi$-band tight-binding model and the classically corrected polarizability (see text), respectively. The only experimental $E_{\mathrm{g}}$ value available is that for the $(15,0)$ nanotube $(0.029 \mathrm{eV})[20]$. 
eV/A. The special $k$-point method plus Gaussian broadening technique was used for the Brillouin zone integration. The calculated nanotube radii are listed in Table 1.

The optical properties were then calculated based on the independent-particle approximation, i.e., the excitonic effects and the local-field corrections were neglected. The imaginary part of the dielectric function $\varepsilon(\omega)$ due to direct interband transitions is given by Fermi golden rule (see, e.g., [18]) (atomic units are used in the rest of this paper), i.e.,

$\varepsilon_{a a}^{\prime \prime}(\omega)=\frac{4 \pi^{2}}{\Omega \omega^{2}} \sum_{i \in \mathrm{VB}, j \in \mathrm{CB}} \sum_{\mathbf{k}} w_{\mathbf{k}}\left|p_{i j}^{a}\right|^{2} \delta\left(\epsilon_{\mathbf{k} j}-\epsilon_{\mathbf{k} i}-\omega\right)$

where $\Omega$ is the unit cell volume and $\omega$ the photon energy. Also, VB and CB denote the conduction and valence bands, respectively. The dipole transition matrix elements $p_{i j}^{a}=\left\langle\mathbf{k} j\left|\hat{p}_{a}\right| \mathbf{k} i\right\rangle$ were obtained from the self-consistent band structures within the PAW formalism [19]. Here $|\mathbf{k} n\rangle$ is the $n$th Bloch state wave function with crystal momentum $\mathbf{k}$ and $a$ denotes the Cartesian components. The real part of the dielectric function is obtained from $\varepsilon^{\prime \prime}(\omega)$ by a Kramer-Kronig transformation

$\varepsilon^{\prime}(\omega)=1+\frac{4}{\pi} \mathbf{P} \int_{0}^{\infty} d \omega^{\prime} \frac{\omega^{\prime} \varepsilon^{\prime \prime}\left(\omega^{\prime}\right)}{\omega^{\prime 2}-\omega^{2}}$.

Here $\mathbf{P}$ denotes the principal value of the integral. Given the complex dielectric function $\left(\varepsilon^{\prime}+i \varepsilon^{\prime \prime}\right)$, all other optical properties such as refractive index, reflectivity and absorption spectrum can be calculated. Furthermore, the electric polarizability $\alpha$ is given by $\varepsilon^{\prime}(\omega)=1+4 \pi \alpha(\omega) / \Omega$. In the present calculations, the $\delta$-function in Eq. (1) is approximated by a Gaussian function with $\Gamma=0.2 \mathrm{eV}$. Furthermore, to ensure that $\varepsilon^{\prime}$ calculated via Kramer-Kronig transformation Eq. (2) are reliable, at least ten energy bands per atom are included in the present optical calculations. Further details can be found elsewhere [15]. In this paper, we are concerned only with the static dielectric constant $\varepsilon^{\prime}(0)$ and electric polarizability $\alpha(0)$.

\section{Results and discussion}

The calculated static dielectric constant $\varepsilon(0)$ and polarizability $\alpha(0)$ as well as band gap $E_{\mathrm{g}}$ of the nanotubes studied are listed in Table 1. Also listed in Table 1 are the band gaps predicted by simple $\pi$ orbital tight-binding model $\left(E_{\mathrm{g}}^{\mathrm{TB}}=2 \pi t / \sqrt{3}(2 n+\right.$ $m), t=2.7 \mathrm{eV}$ and $n-m \neq 3 \times$ integer) for comparison. For metallic nanotubes, the static dielectric constant and polarizability for electric field parallel to the tube axis $(E \| \hat{z})$ are infinite and thus not listed. Table 1 shows that both $\varepsilon(0)$ and $\alpha(0)$ are highly anisotropic, as can perhaps be expected from the one-dimensional character of the nanotubes. The $\varepsilon(0)$ and $\alpha(0)$ for $E \| \hat{z}$ are two to three times larger than that for electric field perpendicular to the tube axis $(E \perp \hat{z})$ (Table 1). Interestingly, $\alpha(0)$ per unit length for both electric field polarizations is roughly proportional to square of tube radius $R^{2}$ (see Fig. 1), i.e., $\alpha(0)=a_{0}+a_{1} R^{2}$, being independent of the chirality and electronic structure of the nanotubes. For $E \perp \hat{z}, a_{0}=10.1$ $\AA^{2}$ and $a_{1}=2.44$ and for $E \| \hat{z}, a_{0}=38.0 \AA^{2}$ and $a_{1}=6.92$. Since the number of atoms per unit on a nanotube is proportional to the tube radius, this indicates that the polarizability of the nanotubes increases roughly with its tube radius. This behavior of $\alpha(0)$ could be understood in an empty lattice model of electrons moving freely on a cylinder of infinitesimal thickness [21]. For $E \perp \hat{z}$, this behavior has also been found in previous semiemprical tight-binding calculations [12]. However, for $E \| \hat{z}, \alpha(0)$ was previously found to be proportional to $R / E_{\mathrm{g}}^{2}$, where $E_{\mathrm{g}}$ is the band gap. In contrast, we find that the present results of $\alpha(0)$ do not follow this relation. This marked difference between previous and present calculations might be attributed to the pronounced differences in the calculated band gaps (Table 1).

For $E \| \hat{z}$, the calculated absorption spectra $[14,15]$ of $4 \AA$ nanotubes $[(5,0),(4,2),(3,3)]$ are in good agreement with the observed absorption spectra [22]. However, the experiments [22] indicate that for $E \perp \hat{z}$, the measured absorption is small and featureless, being very different from the calculated spectra $[14,15]$. This pronounced difference is caused by the local field (depolarization) effects $[10,12]$ which is neglected in 

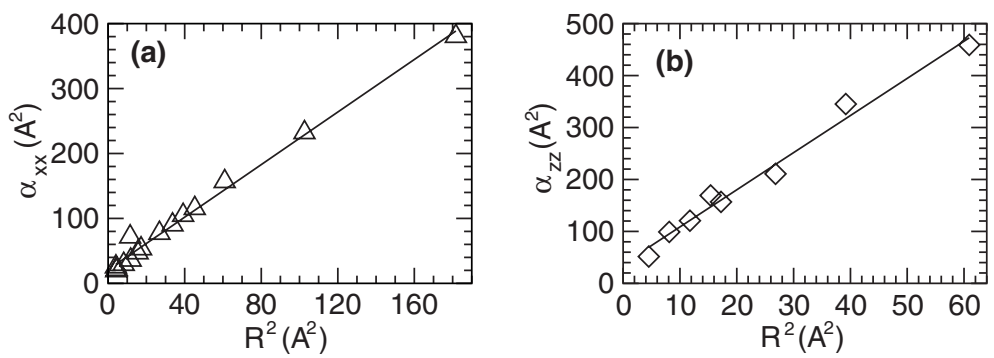

Fig. 1. (a) $\alpha_{x x}(0)$ and (b) $\alpha_{z z}(0)$ vs $R^{2}$ for the nanotubes studied. The solid line is a linear least squares fit.

previous and present ab initio calculations $[14,15]$. When $E \perp \hat{z}$, bound charge will build up on the surface of a nanotube and create a local depolarization field. As a result, the polarizability for $E \perp \hat{z}$ will be reduced. Therefore, the calculated dielectric constant and polarizability from independent-particle approximation for $E \perp \hat{z}$ cannot be compared with experiments directly. In contrast, because the nanotube is infinitely long or long, there will be either no or tiny bound surface charge when $E \| \hat{z}$. Therefore, depolarization has no effect on the dielectric constant and polarizability for $E \| \hat{z}$. Calculation of full interacting many-electron polarizability is beyond the scope of this paper. Nevertheless, as in previous tight-binding calculation [12], we adopt a classical correction to estimate the screened polarizability, i.e.,

$\alpha_{x x}^{\mathrm{cor}}(0)=\frac{\alpha_{x x}(0)}{1+2 \frac{\alpha_{x x}(0)}{R_{\mathrm{eff}}^{2}}}$

where $R_{\text {eff }}=R+\delta R, \delta R=1.2 \AA$ and $R$ is the radius of the nanotube. The depolarization field corrected polarizability $\alpha_{x x}^{\text {cor }}(0)$ are listed in Table 1. Clearly, the unscreened polarizability $\alpha_{x x}^{\text {cor }}(0)$ is reduced by a factor of 4-5 when the classical correction is made. This considerably enhances the anisotropy in the electric polarizability which is now increased up to a factor of 15 .

\section{Acknowledgements}

The authors thank the financial supports from National Science Council of Taiwan (NSC 92-
2120-M002-003) and Ministry of Economic Affairs of Taiwan (MOEA 92-EC-17-A-08-S1-0006).

\section{References}

[1] S. Iijima, Nature (London) 354 (1991) 56.

[2] M. Bockrath, D.H. Cobden, J. Lu, A.G. Rinzler, R.E. Smalley, L. Balents, P.L. McEuen, Nature (London) 397 (1999) 598.

[3] M.F. Lin, D.S. Chuu, Phys. Rev. B 57 (1998) 6731.

[4] L. Liu, G.Y. Guo, C.S. Jayanthi, S.Y. Wu, Phys. Rev. Lett. 88 (2002) 217206.

[5] R. Saito, G. Dresselhaus, M.S. Dresselhaus, Physical Properties of Carbon Nanotubes, Imperial College, London, 1998.

[6] S. Tasaki, K. Maekawa, T. Yamabe, Phys. Rev. B 57 (1998) 9301.

[7] I. Bozovic, N. Bozovic, M. Damnjanovic, Phys. Rev. B 62 (2000) 6971.

[8] O.E. Alon, V. Averbukh, N. Moiseyev, Phys. Rev. Lett. 85 (2000) 5218

[9] E.L. Ivchenko, B. Spivak, Phys. Rev. B 66 (2002) 155404.

[10] H. Ajiki, T. Ando, Physica B 201 (1994) 349; H. Ajiki, T. Ando, Jpn. J. Appl. Phys., Suppl. 34 (1995) 107.

[11] M.F. Lin, K.W.-K. Shung, Phys. Rev. B 50 (1994) 17744.

[12] L.X. Benedict, S.G. Louie, M.L. Cohen, Phys. Rev. B 52 (1995) 8541.

[13] H.J. Liu, C.T. Chan, Phys. Rev. B 66 (2002) 115416.

[14] M. Machon, S. Reich, C. Thomsen, D. Sanchez-Portal, P. Ordejon, Phys. Rev. B 66 (2002) 155410.

[15] G.Y. Guo, K.C. Chu, D.S. Wang, C.-G. Duan, Phys. Rev. B 69 (2004) 205416.

[16] P.E. Blöchl, Phys. Rev. B 50 (1994) 17953; G. Kresse, J. Joubert, Phys. Rev. B 59 (1999) 1758.

[17] G. Kresse, J. Hafner, Phys. Rev. B 47 (1993) 558; G. Kresse, J. Hafner, Phys. Rev. B 49 (1994) 14251; G. Kresse, J. Furthmüller, Comput. Mater. Sci. 6 (1996) 15.

[18] J. Li, C.-G. Duan, Z.-Q. Gu, D.-S. Wang, Phys. Rev. B 57 (1998) 2222. 
[19] B. Adolph, J. Furthmüller, F. Bechstedt, Phys. Rev. B 63 (2001) 125108.

[20] M. Ouyang, J.-L. Huang, C.-L. Cheung, C.M. Lieber, Science 292 (2001) 702.
[21] M.F. Lin, K.W.-K. Shung, Phys. Rev. B 47 (1993) 6617.

[22] Z.M. Li, Z.K. Tang, H.J. Liu, N. Wang, C.T. Chan, R. Saito, S. Okada, G.P. Li, J.S. Chen, N. Nagasawa, S. Tsuda, Phys. Rev. Lett. 87 (2001) 127401. 